\title{
Design of one-way wavefield extrapolation operators, using smooth functions in WLSQ optimization
}

\author{
Jan W. Thorbecke*, Kees Wapenaar*, and Gerd Swinnen*
}

\begin{abstract}
Many depth migration methods use one-way frequency-space depth extrapolation methods. These methods are generally considered to be expensive, so it is important to find the most efficient way of implementing them. This usually means making spatial convolution operators that are as short as possible. Applying the extrapolation operators in a recursive way, using small depth steps, also demands that the operators do not amplify the wavefield at every depth step.

Weighted least squares is an appropriate method to use for designing extrapolation operators that are accurate and efficient and that remain stable in a recursive algorithm. The extrapolated wavefields calculated with these operators are comparable with the extrapolation results obtained with other known operator design techniques as the Remez exchange method and nonlinear optimization. In this paper, the weighted leastsquares technique is refined by using different model functions. By smoothing the phase and amplitude transition at the evanescent cutoff, we can stabilize the resulting operators.

The accuracy of the operators is shown in zero-offset migration impulse responses in $2 \mathrm{D}$ and $3 \mathrm{D}$ media. The Sigsbee2A data set is used to illustrate the quality of the extrapolation operators in prestack depth migration in a complex medium.
\end{abstract}

\section{INTRODUCTION}

Recursive wavefield extrapolation in the space-frequency domain has a number of advantages over other wavefield extrapolation methods, such as phase shift plus interpolation (PSPI) (Gazdag and Sguazzero, 1984), and methods based on the parabolic wave equation (Claerbout, 1985). The most important advantage is extrapolation of one-way wavefields accu- rately through strongly laterally varying media. The disadvantage is that the spatial convolution process is computationally expensive; hence, short convolution lengths are desired to reduce computation costs.

Spatial convolution operators must extrapolate wavefields for high propagation angles in heterogeneous media. Propagation for high angles means that the spatial length of the convolution operator must be large enough to contain those angles. On the other hand, extrapolation in heterogeneous media requires a short spatial operator to handle the lateral variations accurately. These two requirements are in conflict with each other, which explains the need for extrapolation operator optimization. A third requirement is related to the amplitudes of the operator. Because of the recursive use of the operators, the amplitudes must be treated with special care. An amplitude larger than one (in the wavenumber domain) can lead to unstable extrapolation results, while an amplitude smaller than one will usually attenuate the wavefield during extrapolation. Last, but not least, the phase of the operator should be accurate for the whole range of angles for which the operator is designed.

There are many ways to calculate space-variant spatial convolution operators for wavefield extrapolation. The most common approach is to start with the exact analytical expression of the phase-shift operator in the wavenumber-frequency domain and transform this operator back to the spatial domain in some optimum sense. In recent years different methods have been developed to do this transformation in an efficient and optimum way. Holberg (1988) for 2D media and Blacquière et al. (1989) for 3D media use a nonlinear least-squares algorithm with constraints to inverse transform from the wavenumber to the spatial domain. A nonlinear algorithm is usually expensive, will not always find the optimum solution, and can be trapped in a local minimum.

For 2D wavefield extrapolation, Blacquière (1989) uses a phase-shift operator with a smooth phase in the wavenumber domain and inverse Fourier transforms this operator to the spatial domain. There the operator is truncated to a small number of spatial samples to obtain the convolution operator. The number of points to be used in the spatial domain is relatively

Published on Geophysics Online, March 31, 2004. Manuscript received by the Editor July 4, 2003; revised manuscript received December 15, 2003. ${ }^{*}$ Delft University of Technology, Section of Applied Geophysics, Delft, Netherlands. E-mail: j.w.thorbecke@citg.tudelft.nl; c.p.a.wapenaar@ citg.tudelft.nl; g.swinnen@citg.tudelft.nl.

(C) 2004 Society of Exploration Geophysicists. All rights reserved. 
large to remain stable in a recursive extrapolation algorithm. Nautiyal et al. (1993) does not make the phase smoother but uses a Gaussian taper function to smooth the spatial wavelet represented by a Hankel function, for 2D downward extrapolation. Other tapers could also be used, but it can be shown that the Gaussian taper yields a stable extrapolation operator. The disadvantage of this method is that the extrapolation result is only accurate for small propagation angles. Other tapers, such as the Hanning, preserve more of the spatial wavelet character than the Gaussian does but are not guaranteed to lead to stable operators.

Hale's (1991b) method is based on a Taylor series expansion of the phase-shift operator and is stable [excluding the extreme velocity distributions mentioned by Etgen (1994)]. This method uses only a limited number of points, but it cannot handle high angles of propagation. The method used by Soubaras (1992) is based on the $L_{\infty}$ norm and produces extrapolation operators which have an equiripple behavior in the wavenumber domain. Although this method produces quite accurate operators for high angles, the equirippleness of the operators is not strictly needed in wavefield extrapolation, and further improvements can be achieved using a different norm.

In this paper, an improved weighted least-squares (WLSQ) method is presented for an efficient transformation from a phase-shift operator in the wavenumber domain to an accurate convolution operator in the spatial domain. Before discussing this new method, the problems of extrapolation operator optimization are briefly explained. The WLSQ method is then derived from the Fourier integral, and it is shown how the method can be used in extrapolation operator design (Thorbecke and Rietveld, 1994). Furthermore, special attention is paid to the choice of the model function in the WLSQ method and the impact it has on the accuracy of the operator. We show that the choice for smooth model functions in WLSQ optimization leads to significantly more accurate operators than any of the methods described in the literature to date.

The WLSQ method with smooth model functions can easily be extended to $3 \mathrm{D}$ media, and a simple example illustrates the accuracy of the 3D extrapolation operators.

\section{EXTRAPOLATION OPERATORS}

Wavefield extrapolation in the space-frequency domain propagates data from depth level $z_{m}$ to level $z_{m+1}$, where $\Delta z=\left|z_{m+1}-z_{m}\right|$ is small compared to the spatial length of the operator. In a homogeneous layer, the forward one-way extrapolation operator in the $k_{x}, k_{y}-\omega$ (wavenumber-frequency) domain is a well-known analytical function, called the phase-shift operator (Gazdag, 1978), and is given by

$$
\tilde{W}\left(k_{x}, k_{y}, \omega, \Delta z\right)=\exp \left(-j k_{z} \Delta z\right)
$$

where

$$
k_{z}=\left\{\begin{array}{lll}
\sqrt{k^{2}-\left(k_{x}^{2}+k_{y}^{2}\right)} & \text { for } & k_{x}^{2}+k_{y}^{2} \leq k^{2}, \\
-j \sqrt{\left(k_{x}^{2}+k_{y}^{2}\right)-k^{2}} & \text { for } & k_{x}^{2}+k_{y}^{2}>k^{2},
\end{array}\right.
$$

with $k$ defined as $\omega / c, \Delta z$ is a small extrapolation step, $c$ is the propagation velocity of the layer, $j$ is the imaginary unit, and $\omega$ is the angular frequency. Note that for $\left(k_{x}^{2}+k_{y}^{2}\right)>k^{2}$, the wavefield becomes evanescent (i.e., exponentially decaying). The extrapolation result at depth level $z_{m+1}$ can be obtained by multiplying the data at $z_{m}$ with the phase-shift operator in the $k_{x}, k_{y}-\omega$ domain. For laterally varying media, the multiplication in the wavenumber domain by equation (1) is not valid. Instead a space-variant spatial convolution operator in the $x, y-\omega$ (space-frequency) domain can be used to extrapolate the data more accurately. Figure 1 explains the basic concepts of recursive wavefield extrapolation with space-variant spatial convolution operators. At every lateral position another operator is used, based on the local velocity, to extrapolate the data from one depth level to another.

In the $k_{x}-\omega$ domain the extrapolation operator for a $2 \mathrm{D}$ medium is given by equation (1) with $k_{y}=0$ :

$$
\tilde{W}\left(k_{x}, \omega, \Delta z\right)=\exp \left(-j k_{z} \Delta z\right) .
$$

The analytical inverse Fourier transform of equation (3) is a scaled Hankel function (see Berkhout, 1984):

$$
W(x, \omega, \Delta z)=-j k \frac{\Delta z}{2 r} H_{1}^{(2)}(k r),
$$

where the distance $r=\sqrt{\left(x^{2}+\Delta z^{2}\right)} ; \quad H_{1}^{(2)}(k r)=J_{1}(k r)-$ $j Y_{1}(k r)$ is the first-order Hankel function of the second kind; and $J_{1}$ and $Y_{1}$ are the first-order Bessel functions of the first and second kind, respectively. The cheapest way of obtaining a short operator in the space domain is by discretizing equation (4) and truncating it to a finite number of points. The accuracy of the resulting short operator can be assessed by comparing its spectrum with equation (3).

In Figure 2a the amplitude of the wavenumber spectrum of the operator of equation (4), truncated to 25 points, is shown together with the amplitude of the phase-shift operator $\tilde{W}\left(k_{x}, \omega, \Delta z\right)$ (solid line). The wavenumber spectrum of the truncated operator is, in places, significantly larger than one for $\left|k_{x}\right| \leq k$. Recursive application of this operator causes waves to amplify at every extrapolation step, which in the end blows up the extrapolation result.

A shorter operator has even higher bump(s) above amplitude 1.0 in the wavenumber domain and also limits the maximum propagation angle of the extrapolated wavefield more severely. In the limit, a one-point operator can only propagate
Figure 1. The principle of recursive extrapolation with space-variant spatial convolution operators. At every lateral position which has a different velocity, a new operator is used to extrapolate the data from one depth level to another. Within the operator length the medium is assumed to be homogeneous. It is important, therefore, to use the shortest operator possible while minimizing instability.

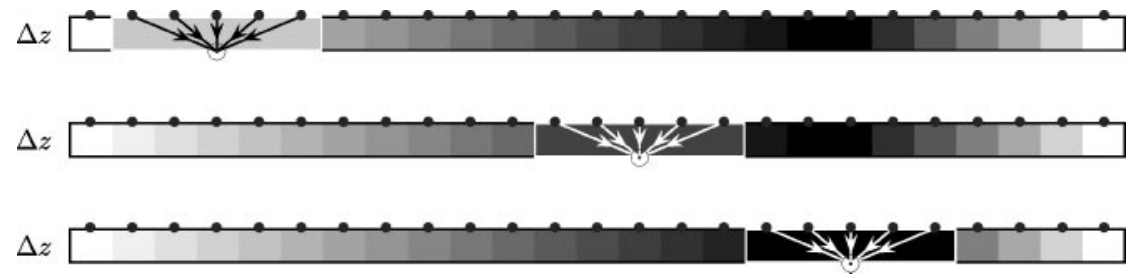


the plane wave corresponding to $k_{x}=0$. The optimal extrapolation operator we are looking for propagates a large range of wave angles accurately and, in a stable sense, consists of only a limited number of points for efficiency. Note that since extrapolation is always done for a finite (but large) number of steps, amplitudes slightly larger than one are allowed. We consider an operator to be stable when its amplitude is smaller than 1.001 for all wavenumbers. In a homogeneous medium and a single frequency, this leads to, after 500 extrapolation steps, a maximum amplification of 1.65 . In reality this factor is usually closer to 1.0 since the extrapolation operators will not be used in a homogeneous medium and the positive maximum errors will not accumulate.

Figure $2 \mathrm{~b}$ shows an operator that has been optimized using the Remez exchange algorithm (Soubaras, 1992). Figures 2c and $2 \mathrm{~d}$ are the result of WLSQ optimization (discussed following). Note that the amplitude error for the WLSQ operator, as shown in Figure 2d, is 0.0001 and gives a maximum error of $5 \%$ after 500 extrapolation steps.

\section{WEIGHTED LEAST SQUARES}

The goal in the optimization procedure is to obtain a short spatial convolution operator with a wavenumber spectrum that is equal or close to the exact formulation in the frequencywavenumber domain over a desired wavenumber band. This problem can be written in general terms as an integral equation based on the spatial Fourier transform:

$$
\begin{aligned}
\tilde{W}\left(k_{x}, \omega, \Delta z\right)= & \int_{x_{1}}^{x_{2}} \exp \left(j k_{x} x\right) W(x, \omega, \Delta z) \mathrm{d} x \\
& \text { for } \quad k_{x, 1} \leq k_{x} \leq k_{x, 2},
\end{aligned}
$$

where $W(x, \omega, \Delta z)$ is the convolution operator to be determined. For symmetric operators and in the remainder of this paper, $x_{2}=-x_{1}$ and $k_{x, 2}=-k_{x, 1}$. Integration is carried out over a limited spatial interval representing the short operator, and the frequency-wavenumber domain of the operator is band limited. The discrete counterpart of the integral in equation (5)

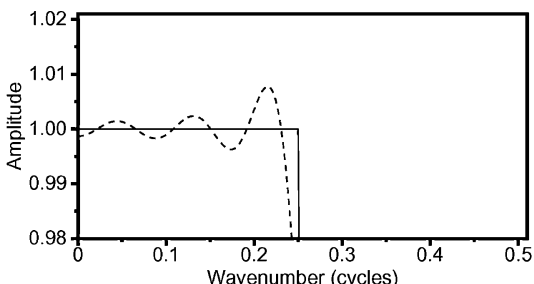

a)

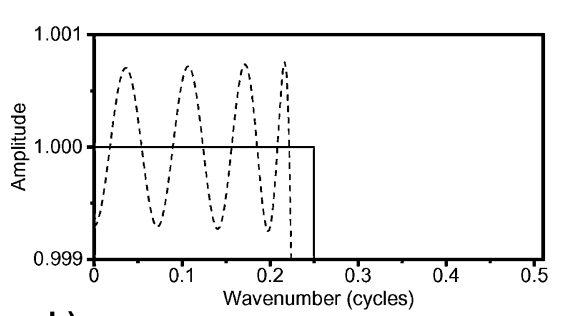

b)

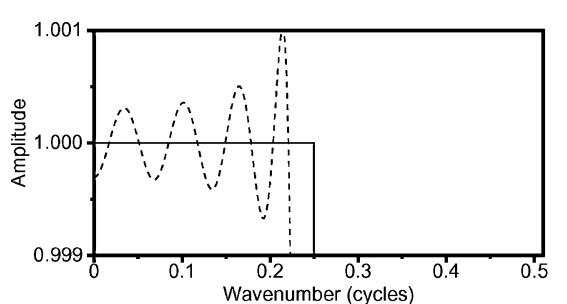

c)

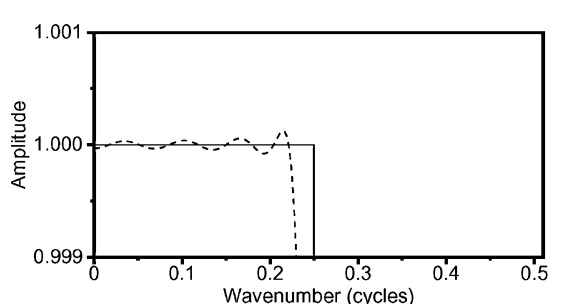

d)
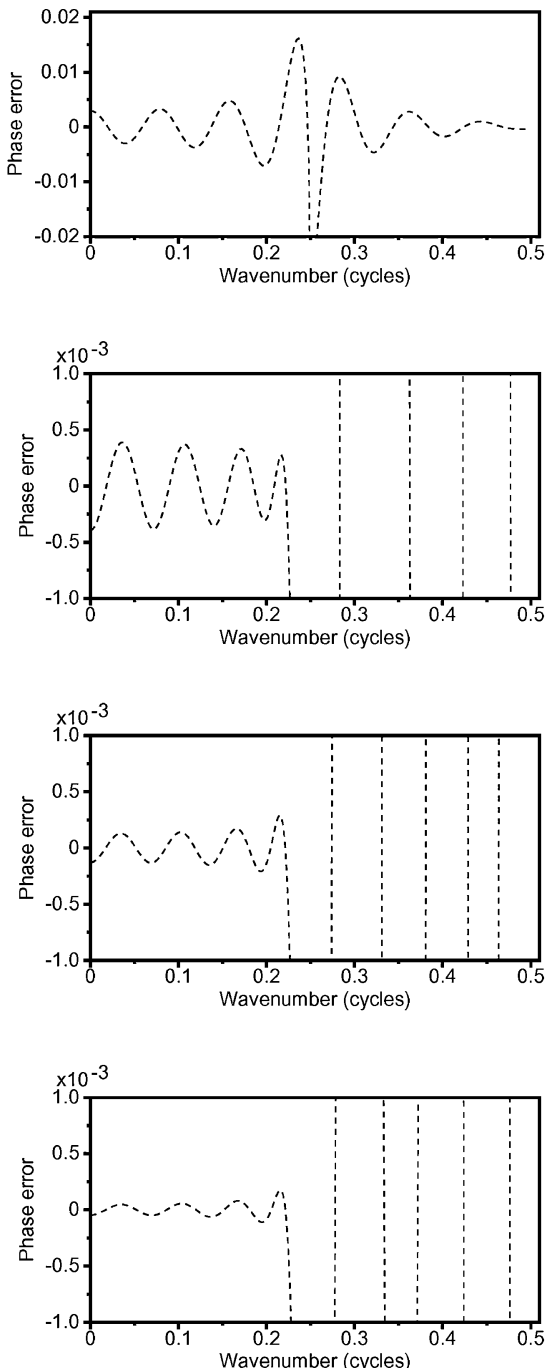

Figure 2. Comparison between spectra of various wavefield extrapolation operators. Amplitude is left column; phase error is right column. For each operator, $k=\omega / c=\pi / 2 \Delta x$. Since the operators are symmetric, only the right half is shown. (a) The effect of the truncation of the analytical operator as seen in the wavenumber amplitude and phase spectrum. (b) The result after optimization with the Remez exchange algorithm. (c) WLSQ optimization with the model function $\tilde{\mathbf{w}}$ being equal to the phase-shift operator. (d) Using a smooth version of $\tilde{\mathbf{w}}$ given by equation (14b). Note the vertical scale difference between (a) and (b)-(d). For all these figures, we have chosen a full operator length of 25 points $(M=12), \Delta x=10 \mathrm{~m}, \Delta z=2 \mathrm{~m}, \omega=50 \pi \mathrm{ra}-$ dians $/ \mathrm{s}, N=512$ samples, $c=1000 \mathrm{~m} / \mathrm{s}$, and a maximum propagation angle of $\alpha_{\max }=75^{\circ}$. The horizontal axis represents normalized wavenumber cycles $\left(k_{x} \Delta x / 2 \pi\right)$. 
is (omitting $\omega$ and $\Delta z$ in the arguments of $\tilde{W}$ and $W$ )

$$
\begin{gathered}
\tilde{W}\left(n \Delta k_{x}\right)=\Delta x \sum_{m=-M}^{M} \exp \left(j n \Delta k_{x} m \Delta x\right) W(m \Delta x) \\
\text { for } \quad-N \leq n \leq N .
\end{gathered}
$$

Written explicitly in matrix notation, the discrete representation of equation (5) is

$$
\left[\begin{array}{c}
\tilde{W}\left(-N \Delta k_{x}\right) \\
\vdots \\
\tilde{W}(0) \\
\vdots \\
\tilde{W}\left(N \Delta k_{x}\right)
\end{array}\right]=\left[\begin{array}{c}
\exp \left(j N \Delta k_{x} M \Delta x\right) \\
\vdots \\
1 \\
\vdots \\
\exp \left(-j N \Delta k_{x} M \Delta x\right)
\end{array}\right.
$$$$
\begin{array}{ccc}
\ldots & 1 & \ldots \\
\ddots & \vdots & \ldots \\
\ldots & 1 & \ldots \\
\ldots & \vdots & \\
\ldots & 1 & \ldots
\end{array}
$$

or

$$
\tilde{\mathbf{w}}=\Gamma \mathbf{w} .
$$

Here, w represents the short operator and $\tilde{\mathbf{w}}$ is its spatial Fourier transform, yielding an approximation of the exact phase-shift operator. Further, $m=-M, \ldots, M$ represents the samples of the short operator, and $n=-N, \ldots, N$ represents the samples of the Fourier transform. Wavenumber sampling is given by

$$
\Delta k_{x}=\frac{2 \pi}{(2 N+1) \Delta x},
$$

and the number of samples in the frequency-wavenumber domain $(2 N+1)$ is chosen such that $2 N+1 \geq 2 M+1$. Note that only $M+1$ independent coefficients have to be computed to construct the full $2 M+1$ convolution operator. In this paper convolution operator sizes are indicated by their full length.

With respect to the unknown spatial operator w, equation (7) has more equations than unknowns. To solve this problem, the weighted prediction error function (Menke, 1989, p. 54) is used

$$
\tilde{\varepsilon}=\tilde{\mathbf{e}}^{H} \tilde{\Lambda} \tilde{\mathbf{e}},
$$

where superscript $H$ denotes a complex-conjugate transpose and

$$
\tilde{\mathbf{e}}=\Gamma\langle\mathbf{w}\rangle-\tilde{\mathbf{w}} .
$$

Diagonal matrix $\tilde{\Lambda}$ contains a weighting function on its diagonal, and $\boldsymbol{\Gamma}\langle\mathbf{w}\rangle$ represents an approximation of $\tilde{\mathbf{w}}$. The weighting function is defined in such a way that the wavenumber components in the propagating part of the operator, up to a chosen maximum angle, are given a relatively high weight. The wavenumber components above the maximum angle are given a low weight factor. The least-squares solution of equation (7) using equation (9) is given by

$$
\langle\mathbf{w}\rangle=\left[\Gamma^{H} \tilde{\Lambda} \Gamma\right]^{-1} \Gamma^{H} \tilde{\Lambda} \tilde{\mathbf{w}},
$$

where $\langle\mathbf{w}\rangle$ is the least-squares approximation of $\mathbf{w}$. The components of the Fourier transform matrix are

$$
\Gamma_{n m}=\exp \left(j n \Delta k_{x} m \Delta x\right),
$$

and the components of the diagonal weighting matrix are given by

$$
\Lambda_{n m}=w\left(n \Delta k_{x}\right) \delta_{n m},
$$

$w$ being a box-shaped weighting function. The matrix that must be inverted, $\Gamma^{H} \tilde{\Lambda} \boldsymbol{\Gamma}$, is a square $M \times M$ matrix. For $1 \mathrm{D}$ operators this matrix has a Toeplitz structure and can be inverted

$$
\left.\begin{array}{lc}
\ldots & \exp \left(-j N \Delta k_{x} M \Delta x\right) \\
\ldots & \vdots \\
\ldots & 1 \\
\ddots & \vdots \\
\ldots & \exp \left(j N \Delta k_{x} M \Delta x\right)
\end{array}\right]\left[\begin{array}{c}
\Delta x W(-M \Delta x) \\
\vdots \\
\Delta x W(0) \\
\vdots \\
\Delta x W(M \Delta x)
\end{array}\right]
$$

efficiently using the Levinson scheme. If in equation (11) the weight matrix is chosen identical to the unit matrix $\boldsymbol{\Lambda}=\mathbf{I}$, then the right-hand side of equation (11) is an inverse Fourier transform of $2 N+1$ points, which is truncated to $2 M+1$ points in the spatial domain. In this specific case no optimization is carried out.

\section{MODEL FUNCTION $\tilde{\mathbf{w}}$}

We consider two WLSQ optimization procedures using different choices for the model function $\tilde{\mathbf{w}}$. For the first procedure, the model function $\tilde{\mathbf{w}}$ in equation (10) is chosen to be the exact phase-shift operator. Note that the phase-shift operator has sharp edges at $k_{x}= \pm k$, which can cause problems if one wants to approximate this operator in a least-squares manner.

It is justified to neglect the evanescent part in the extrapolation operator because it is not present in the data above the noise level. The recursive extrapolation scheme only demands a stable behavior of the wavenumber components in the evanescent region; the phase is of no importance. The weighting function is chosen to be a simple box function with a weight of $1.0 \mathrm{in}$ side the domain of interest (the propagating waves) and a small value $\left(10^{-5}\right)$ outside this band. In Figure $2 \mathrm{c}$, the wavenumber spectrum of a WLSQ optimized operator is shown. From Figure $2 \mathrm{c}$ we observe that the wavenumber spectrum is stable (in the sense discussed above) for all wavenumbers and is accurate within the band of interest. The character of the evanescent part in the wavenumber spectrum is not exponentially decaying any more but is varying (not shown in Figure 2c).

For accurate extrapolation results, the desired operator $\tilde{\mathbf{w}}$ must be equal to the phase-shift operator for the propagating waves. Behavior outside this part of the spectrum, however, can differ from the phase-shift operator. For the second procedure, a so-called smooth phase-shift operator has been designed such that outside the band of interest the amplitude and the phase are defined by a cubic spline which goes smoothly to zero:

$$
\begin{aligned}
& \left\|\tilde{W}\left(k_{x}, \omega, \Delta z, \alpha_{\text {max }}\right)\right\| \\
& \quad= \begin{cases}1.0 & \left|k_{x}\right| \leq k \sin \left(\alpha_{\max }\right) \\
\text { spline } & \left|k_{x}\right|>k \sin \left(\alpha_{\max }\right), \\
0 & \left|k_{x}\right|=\frac{\pi}{\Delta x}\end{cases}
\end{aligned}
$$




$$
\begin{aligned}
& \arg \left(\tilde{W}\left(k_{x}, \omega, \Delta z, \alpha_{\max }\right)\right) \\
& \quad= \begin{cases}-j k_{z} \Delta z & \left|k_{x}\right| \leq k \sin \left(\alpha_{\max }\right) \\
\text { spline } & \left|k_{x}\right|>k \sin \left(\alpha_{\max }\right), \\
0 & \left|k_{x}\right|=\frac{\pi}{\Delta x}\end{cases}
\end{aligned}
$$

where $\alpha_{\max }$ is the maximum propagation angle of interest. The weighting function is a simple box function. Using this smooth model function, the least-squares algorithm finds a smoother solution and has significantly lower amplitude oscillations in the wavenumber spectrum. This can be observed by comparing Figure $2 \mathrm{c}$ with Figure 2d. Changing the weighting function shape-for example, with a smooth transition zone between the low and high weight region-will give less accurate or unstable extrapolation operators.

In Figure 3, the frequency-wavenumber spectrum of the amplitude errors of optimized extrapolation operators are shown for a range of wavenumber values. Figure $3 \mathrm{a}$ shows the amplitude errors of operators with the phase-shift operator as model function, and Figure $3 \mathrm{~b}$ is based on the smooth model function. At the edges where $k_{x}= \pm \frac{\omega}{c} \sin \left(\alpha_{\max }\right)$, both operators have their highest amplitude oscillations (Gibb's phenomenon). These oscillations can give rise to small artifacts at high propagation angles. The operators based on the smooth operator (Figure $3 \mathrm{~b}$ ) have lower amplitude errors than those based on the phase-shift operators (Figure 3a) and are therefore more accurate.

The accurate extrapolation operators can also be tailored for anisotropic media (Zhang et al., 2001) or other special purposes. For example, asymmetric operators can be designed and used in areas where only a small number of receivers is available (Swinnen et al., 2001).

\section{MIGRATION RESULTS ON SYNTHETIC DATA}

First we analyze the accuracy of the various operators using impulse responses. Zero-offset migration experiments are done with a 25-point extrapolation operator in a homogeneous medium, a velocity of $2000 \mathrm{~m} / \mathrm{s}$, a receiver spread of $2000 \mathrm{~m}$, and a maximum extrapolation depth of $1000 \mathrm{~m}$ with $\Delta x=10, \Delta z=2 \mathrm{~m}$. The trace in the middle of the unmigrated zero-offset section contains three Ricker wavelets at $0.3,0.6$, and $0.9 \mathrm{~s}$; all other traces contain only zeros. The wavelet is sampled with $4 \mathrm{~ms}$ and has a frequency peak at $30 \mathrm{~Hz}$. The migration is carried out with half the velocity, i.e., $1000 \mathrm{~m} / \mathrm{s}$.

Figure 4a shows the impulse responses for a nonrecursive reference result. Operators designed with the equiripple approach of the Remez algorithm (Soubaras, 1992) are used to obtain the impulse response in Figure 4b. The WLSQ operators based on the phase-shift operator yield the impulse response in Figure 4c, and WLSQ operators based on the smooth phaseshift operator yield the impulse in Figure 4d. The smooth operator result has on average the least numerical artifacts. At the higher angles (above $75^{\circ}$ ) more artifacts are introduced. In the difference plots of Figure 5, these artifacts are clearly visible. Both WLSQ methods give a better result than the Remez exchange method.

Note that the WLSQ operator optimization is done for a fixed operator length, a fixed weight factor, and a fixed maximum angle of interest. However, convolution operators for lower $\omega$ values can often be shorter than for higher $\omega$ values. By choosing a smaller weight outside the wavenumber band of interest, a more accurate operator within the desired band is obtained. For a value that is chosen too small, the amplitude can become larger than 1.0 outside the band of interest and cause unstable behavior in the recursive algorithm. The input

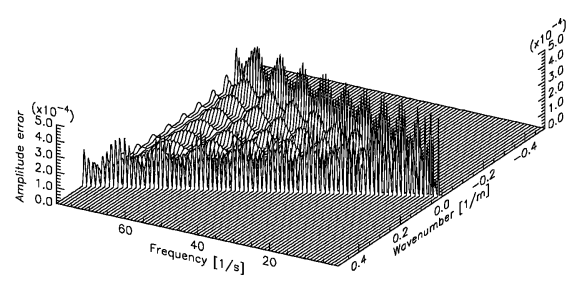

a) WLSQ with phase-shift as model function

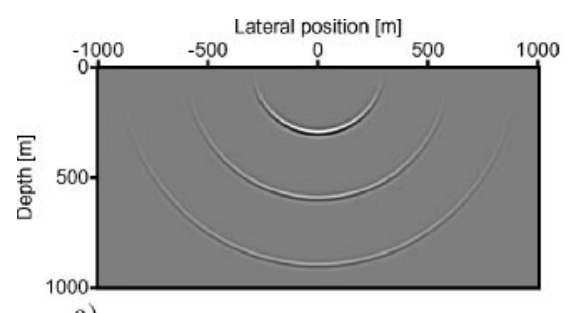

a)

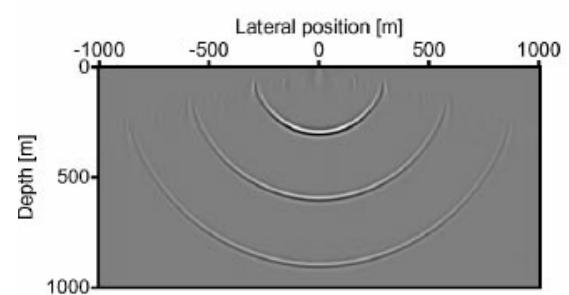

c)

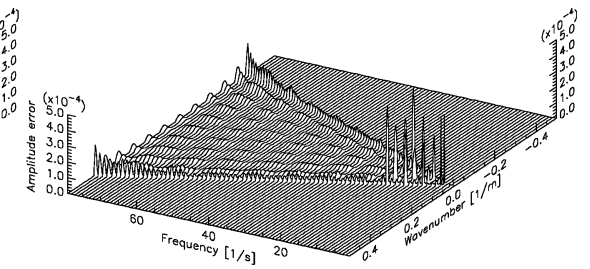

b) WLSQ with smooth phase-shift as model function

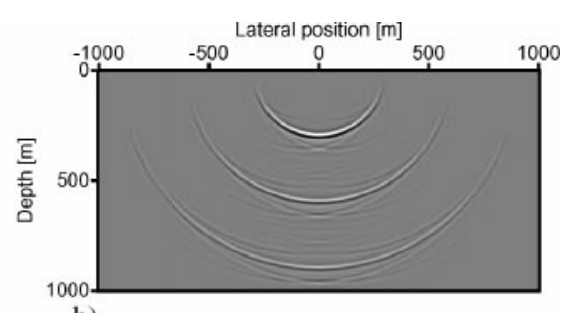

b)

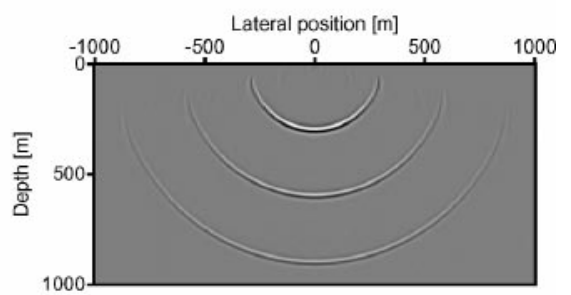

d)

Figure 3. Amplitude errors of extrapolation operators in the frequency-wavenumber domain. The operators are computed for a frequency range of 0 to $80 \mathrm{~Hz}, \Delta t=4 \mathrm{~ms}$, $\Delta x=10 \mathrm{~m}, \Delta z=2 \mathrm{~m}$, velocity of $2000 \mathrm{~m} / \mathrm{s}$ and full operator length of 25 points in the space domain $(M=12)$. The operators based on a smooth phase-shift operator have lower amplitude errors than the ones based on the exact phase-shift operator.

Figure 4. Migration impulse responses for (a) a nonrecursive reference result and for three recursive methods using (b) Remez exchange optimized operators, (c) WLSQ based on phase-shift operator, and (d) WLSQ based on the smooth phase-shift operator. The impulse responses are computed for a frequency range of 0 to $80 \mathrm{~Hz}, \Delta t=4 \mathrm{~ms}, \Delta x=10 \mathrm{~m}$ $\Delta z=2 \mathrm{~m}, \alpha_{\max }=75^{\circ}$, velocity of $2000 \mathrm{~m} / \mathrm{s}$, and full operator length of 25 points. 
parameters of the WLSQ algorithm can be used to search for the shortest, most accurate, and most stable operator within a small range of input parameters (operator size, weight factor, and angle).

The WLSQ algorithm used to calculate the operators is very fast (Levinson recursion) and enables us to do multiple evaluations for the same operator within a short computation time.

\section{Sigsbee2A data set}

As a second experiment we use a synthetic data set to examine the accuracy of a prestack depth migration result using the extrapolation operators based on the smooth phase-shift operator. The synthetic Sigsbee2A data set (Paffenholz et al., 2002) (courtesy of SMAART JV) is based on the geologic setting found on the Sigsbee2A escarpment in the deepwater Gulf of Mexico. The velocity model is shown in Figure 6. The complex salt shape and the high-velocity contrast scatters the wavefield severely. Because not all scattered energy is recorded, illumination problems will occur in the migration.

The Sigsbee2A data set is used to test the accuracy of the extrapolation operators. The used velocity model has a trace increment of $11.4 \mathrm{~m}$, a depth sampling of $7.6 \mathrm{~m}$, and a total depth of $9144 \mathrm{~m} ; 500$ shots were modeled using an acoustic finite-difference scheme for constant density. The source interval is $45.7 \mathrm{~m}$, and the receiver interval is $45.7 \mathrm{~m}$ (original data had a receiver spacing of $22.9 \mathrm{~m}$ ). The minimum offset is $0 \mathrm{~m}$, and the maximum offset is $7932 \mathrm{~m}$ (384 receivers per shot). Starting at the right side of the model, the source location walks through the cable until the full cable length is reached $(7932 \mathrm{~m})$. The dominant frequency of the wavelet is $20 \mathrm{~Hz}$. The record length is 12 seconds, and the sample rate is $8 \mathrm{~ms}$. In the migration, an extra aperture of 500 points $(5715 \mathrm{~m})$ on each side of the acquisition geometry has been used to allow wavefields to propagate outside the acquisition aperture.

The prestack depth migration result shown in Figure 7 has been obtained by using spatial convolution operators of 25 points. For every operator, a search has been carried out to find the weight factor which gives the operator with the smallest amplitude error. Building the operator table for the complete range of velocities and frequencies took less than a minute on a standard PC.

The steep faults beside the salt are imaged correctly. In the zoom area below the salt, all events that contain reflection energy are visible. Close to the right steep bottom of the salt, no layers are visible and it seems that an internal multiple of
Figure 5. The numerical artifacts of the recursive extrapolation, displayed by subtracting the reference result from the recursive results. Note that the pictures are plotted with a 20 times smaller clip value than Figure 4. (a) Nonrecursive reference result, having the same clip value as the other views. (b) Remez equiripple operators. (c) WLSQ with phase-shift operators. (d) WLSQ with smooth operators.

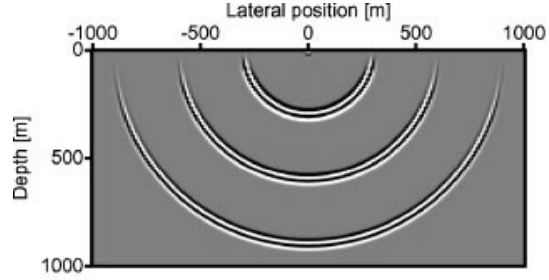

a)

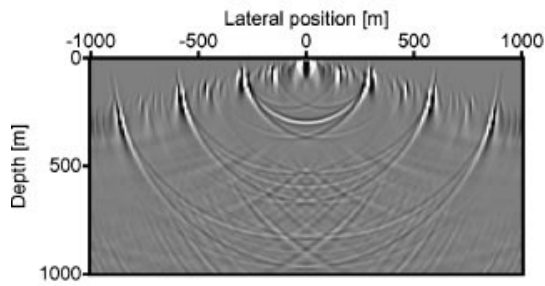

c)

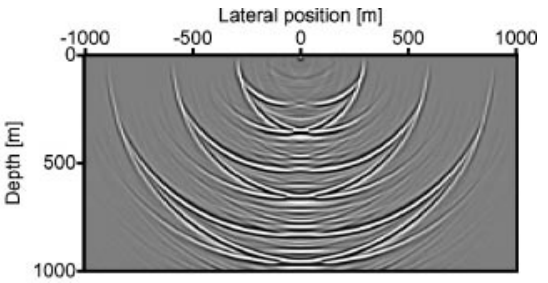

b)

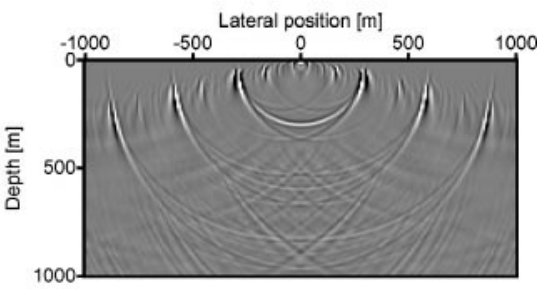

d)

Figure 6. The reflection interfaces are the result of velocity contrasts which fluctuate within $\pm 91 \mathrm{~m} / \mathrm{s}$ relative to a linear velocity function. This velocity contrast is large enough to produce reflections but not sufficient to destroy the basic gradient macromodel used in the depth migration. The salt velocity is $4511 \mathrm{~m} / \mathrm{s}$, and the sediment velocity used in the migration is $V=1524+0.3\left(Z-Z_{\text {seafloor }}\right) \mathrm{m} / \mathrm{s}$.

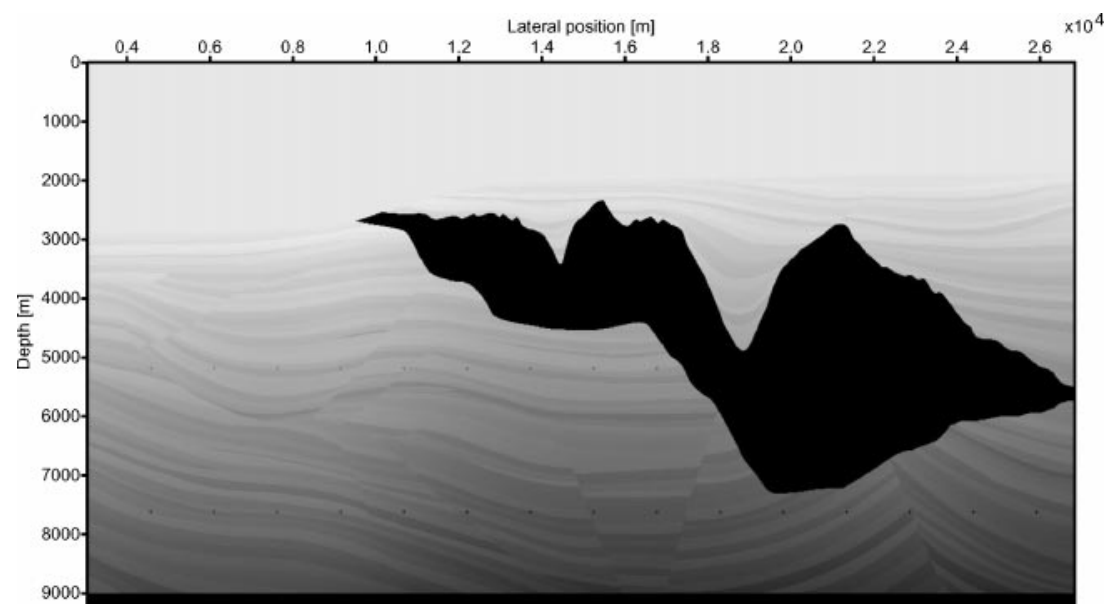


the salt body (indicated by an arrow) has been imaged as a steep ghost fault crossing the layers.

The number of floating-point operations (flops) for the convolution only are related to the spatial extrapolation aperture $A$, the number of depth steps $Z$, and the half-operator length $M+1$ defined by equation (6). The total number of flops per frequency is then given by $((M+1) \times 24+4) \times(A \times Z)$. The 24 represents the flops needed for the complex and symmetric multiplication of both source and receiver wavefields. The four extra flops are used for the imaging condition. Note that the number of flops are not a correct indication for the total single CPU running time. The algorithm used to carry out the 1D convolution is optimized for cache reusage in scalar processors and will also run very efficiently on vector processors, which can handle small vector lengths.

By decreasing the operator length $M$, the run time will also decrease. To make a smaller operator stable, it must be less accurate at high angles. In Figure 8 the imaging result of a seven-point $(M=3)$ operator, designed to be accurate up to $45^{\circ}$, is shown for the same zoom area as in Figure 7. The total run time was decreased by a factor of 2.3. The image quality of Figure 8 is not as good as Figure 7, and the steep base of the salt is missing. But the result with this small seven-point operator is still quite useful.
The disadvantage of recursive one-way migration, as used in this paper, is that it is impossible to handle turning/bending waves. Also, the transmission coefficients are not included, resulting in lower amplitudes. Structurally, the one-way migration method produces accurate images below the salt.

\section{EXTENSION TO 3D EXTRAPOLATION}

The extension of the WLSQ technique for 2D convolution operators to be applied in 3D media is straightforward. The 2D Fourier transformation is defined by

$$
\begin{aligned}
\tilde{W}\left(k_{x}, k_{y}, \omega, \Delta z\right)= & \iint_{-\infty}^{+\infty} W(x, y, \omega, \Delta z) \\
& \times \exp \left(j k_{x} x\right) \exp \left(j k_{y} y\right) \mathrm{d} x \mathrm{~d} y .
\end{aligned}
$$

Using a discrete version of the Fourier integral and the circular symmetry in the phase-shift operator, equation (15) can be rewritten as (Blacquière et al., 1989)

$$
\begin{aligned}
& \tilde{W}\left(p \Delta k_{x}, q \Delta k_{y}\right) \approx \Delta x \Delta y \sum_{m=0}^{M} \sum_{n=0}^{N} S_{m n} W(m \Delta x, n \Delta y) \\
& \quad \times \cos \left(p \Delta k_{x} m \Delta x\right) \cos \left(q \Delta k_{y} n \Delta y\right),
\end{aligned}
$$

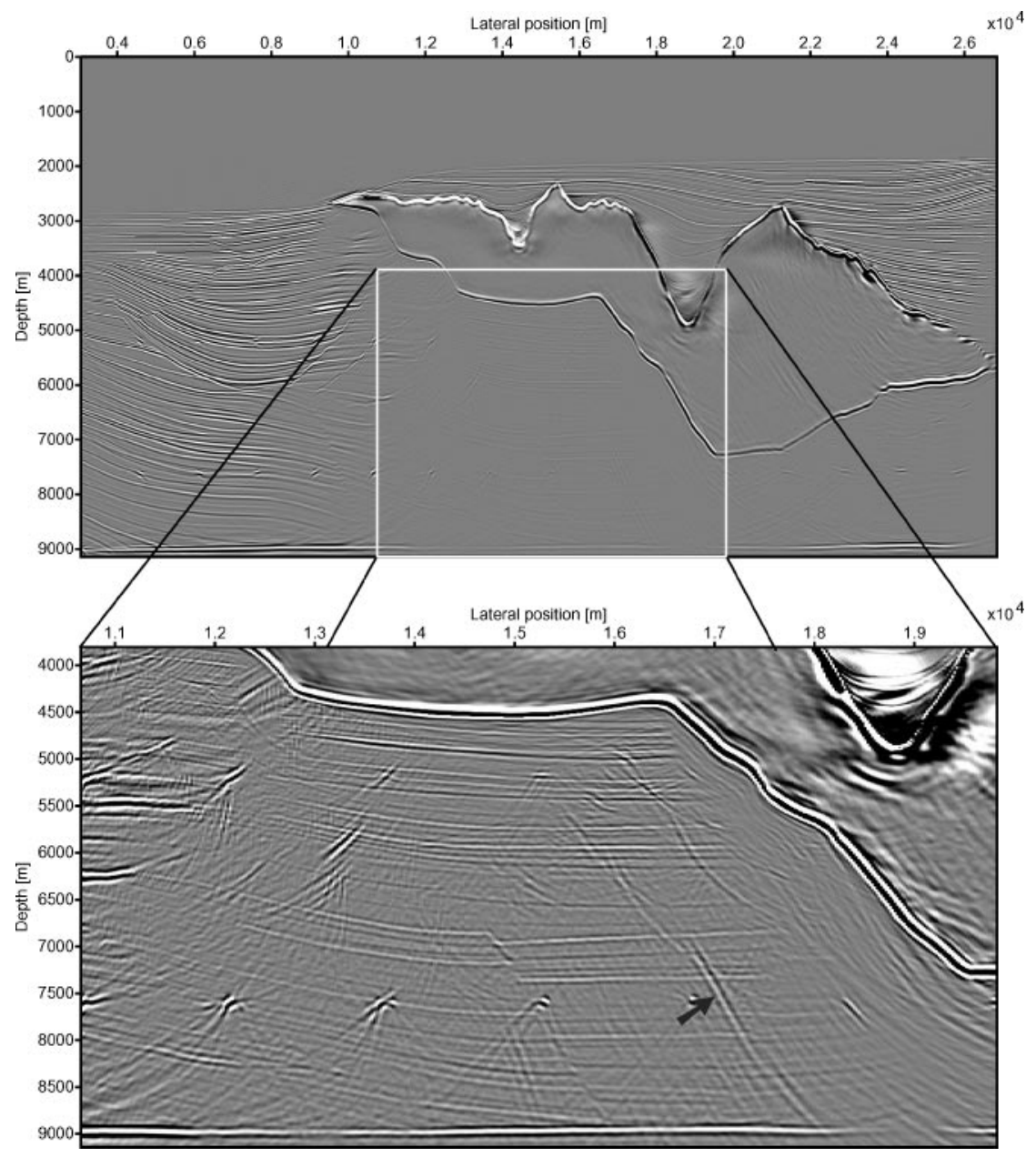

Figure 7. Prestack depth migration of Sigsbee2A data set with optimized WLSQ operators with a fixed length of 25 points based on the smooth model function. The bottom picture is a zoom of the area below the salt. The transmission coefficients through the salt are not taken into account, giving a loweramplitude image below the salt compared with the surrounding areas. Most events below the salt are imaged. Only the turning waves are not imaged. The event indicated by the arrow represents an internal multiple of the salt body, which has been imaged as a steep ghost fault crossing the layers. 
with $S_{m n}$ defined as

$$
S_{m n}=\left\{\begin{array}{lll}
1 & \text { for } & m=n=0, \\
2 & \text { for } & m=0 \vee n=0, \\
4 & \text { for } & n \neq 0 \wedge m \neq 0 .
\end{array}\right.
$$

By interchanging $n$ and $m$ and using the fact that $\sum_{m=0}^{M} \sum_{n=0}^{m}=$ $\sum_{n=0}^{M} \sum_{m=0}^{n}$, the number of unknowns can be further reduced to $1 / 8$ of the original number of unknowns. Note that this reduction is only possible in isotropic media with $\Delta x=\Delta y$. Hence,

$$
\begin{aligned}
\tilde{W}\left(p \Delta k_{x}, q \Delta k_{y}\right) & \approx \Delta x^{2} \sum_{m=0}^{M} \sum_{n=0}^{m} W(m \Delta x, n \Delta y) \\
& \times\left[S_{m n} \cos \left(p \Delta k_{x} m \Delta x\right) \cos \left(q \Delta k_{y} n \Delta y\right)\right. \\
& \left.+S_{n m}^{\prime} \cos \left(p \Delta k_{x} n \Delta x\right) \cos \left(q \Delta k_{y} m \Delta y\right)\right],
\end{aligned}
$$

with $S_{n m}^{\prime}$ defined as

$$
\begin{cases}0 & \text { for } n=m \\ 2 & \text { for } n=0 \vee m=0 \\ 4 & \text { for } m \neq 0 \wedge n \neq 0\end{cases}
$$

and $M \times M$ being the user-specified size of the desired short operator.

Equation (16) or equation (18) must be solved for the unknown operator coefficients $W_{m n}=W(m \Delta x, n \Delta y)$ for all equations. The same weighted error function $\tilde{\varepsilon}$ as introduced in equation (9) is used, and the same least-squares solution is obtained:

$$
\langle\mathbf{w}\rangle=\left[\Gamma^{H} \tilde{\Lambda} \boldsymbol{\Gamma}\right]^{-1} \boldsymbol{\Gamma}^{H} \tilde{\Lambda} \tilde{\mathbf{w}},
$$

where $\Gamma^{H} \tilde{\Lambda} \boldsymbol{\Gamma}$ is a square matrix. For the $1 \mathrm{D}$ optimization problem, the WLSQ method could be inverted using a fast Levinson scheme. For the 2D problem, the Toeplitz structure is not present, and LAPACK routines are used to calculate a QR decomposition of the matrix $\Gamma^{H} \tilde{\Lambda} \Gamma$ in equation (20).

Figure 9 shows the wavenumber spectrum of a WLSQ optimized spatial convolution operator based on a smooth model function of $25 \times 25$ points. The WLSQ method gives an accurate operator which has a wavenumber spectrum close to the exact phase-shift operator. Because of the optimization on a rectangular grid, the operator has a square footprint. The accuracy of the operator is similar to an operator obtained with nonlinear least squares, but the design of the WLSQ algorithm is more efficient.

The operators obtained with this method require the implementation of a full 2D spatial convolution. Using the symmetry in the operator, the number of multiplications in the $2 \mathrm{D}$ convolution can be reduced by a factor of four by folding the data into a quarter and applying the convolution to this folded part only. The number of flops remains high, however, especially if one takes into account that this convolution has to be carried out for every gridpoint, for every frequency of interest, and for all depth steps. Alternative and more efficient recursive extrapolation operators like the Hale-McClellan (Hale, 1991a; McClellan, 1973) operator are discussed in more detail in Appendix A of Thorbecke (1997) and in Thorbecke and Geesink (1999). The 2D WLSQ extrapolation operator can be used very well in a Hale-McClellan scheme.

In Figure 10, a 3D impulse response using WLSQ operators is shown. The top right-hand side in Figure 10 shows a vertical cross-section for $x=0$, and the bottom right-hand side shows a vertical section for $x=y$. The left-hand side shows a horizontal cross-section at a depth of $220 \mathrm{~m}$, which corresponds to a reflector dip of $65^{\circ}$. The impulse response is circular, but the artifacts introduced at high propagation angles are rectangular.

Figure 8. Prestack depth migration below the salt of the Sigsbee2A data set with optimized WLSQ operators with a length of 7 points. Because of the short operator length, not all high angles have been propagated. This is observed in the lack of steeps events compared to Figure 7 and the missing steep bottom of the salt. The clipping value of the plot is chosen the same as in Figure 7.

Figure 9. The wavenumber spectrum of a WLSQ optimized operator with $25 \times 25$ spatial points. The operator has a frequency of $25 \mathrm{~Hz}$ with $c=1000 \mathrm{~m} / \mathrm{s}, \Delta x=\Delta y=10 \mathrm{~m}$, $\Delta z=2 \mathrm{~m}$, and maximum angle of interest set at $75^{\circ}$.
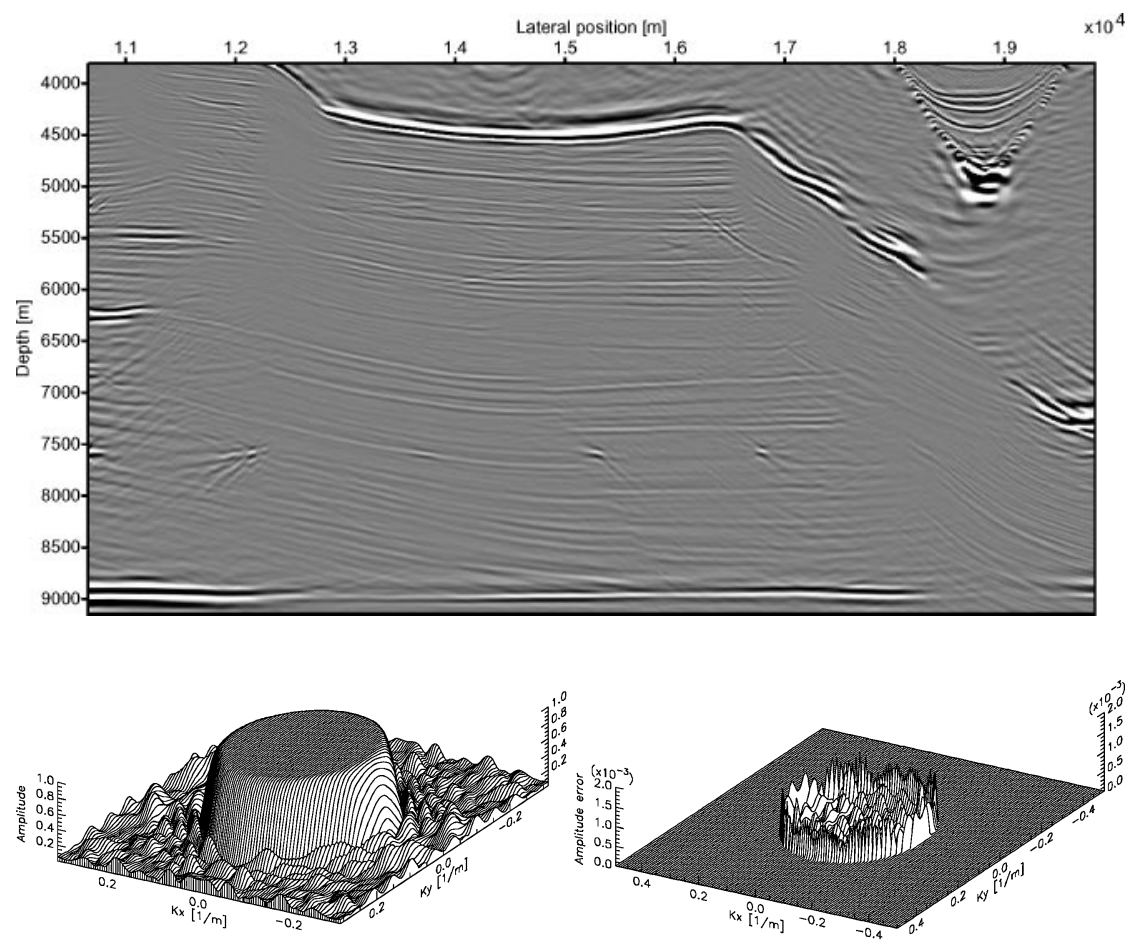

a) Wavenumber spectrum

b) Amplitude error of a) 
a)

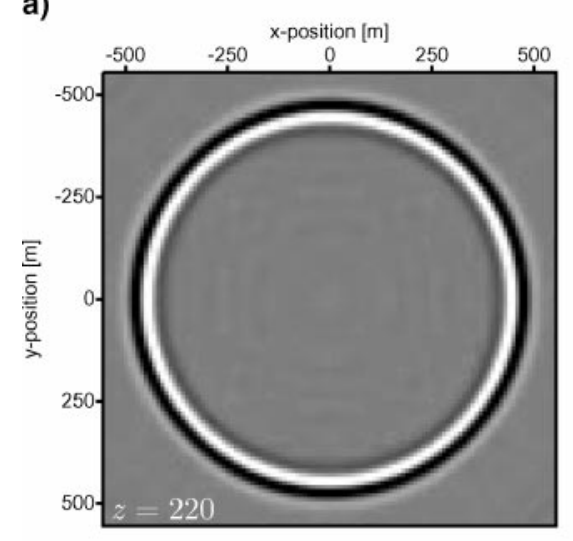

b)
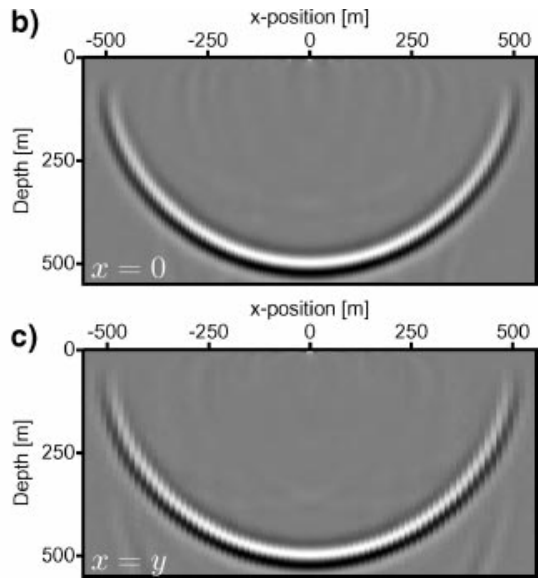

Figure 10. The 3D impulse response of a $25 \times 25$ WLSQ operator. (a) Depth slice at $z=220 \mathrm{~m}$. (b) Vertical slice for $x=0 \mathrm{~m}$. (c) Diagonal slice for $x=y$. Note the circular shape and the accuracy at the higher angles. For this experiment, the velocity $c=1000 \mathrm{~m} / \mathrm{s}$, a frequency range of $(1-45) \mathrm{Hz}, \Delta x=\Delta y=10 \mathrm{~m}$, $\Delta z=2 \mathrm{~m}, \Delta t=0.004 \mathrm{~s}$, and $\alpha_{\max }=75^{\circ}$ on a spatial grid of $111 \times 111$ samples wide. In the diagonal cross-section, the distance between two traces is $\sqrt{2} \Delta x$, where $\Delta x$ is the distance between the traces in the vertical $x=0$ section.

\section{DISCUSSION AND CONCLUSION}

In this paper we have introduced a method to compute convolutional wavefield extrapolation operators that are accurate for high propagation angles and have a short spatial extent. The method is based on WLSQ and uses a smooth transition zone at the evanescent cutoff. The presented results show that these improved operators give very accurate extrapolation results.

The WLSQ algorithm to compute the operators is very efficient. This allows multiple evaluations for different weighting functions and operator lengths, thus searching for the best operator with minimum operator length and smallest amplitude and phase errors.

The WLSQ is not only suited for extrapolation operator design, but it can also be used in other filter design problems, achieving an efficient and controlled transformation of the operator in the Fourier domain to a convolution operator in the original domain.

\section{ACKNOWLEDGMENTS}

The authors would like to thank the Integrated Solid Earth Science (ISES) research school and the EU Atlass project for supporting this research. Associate Editor Tamas Nemeth and reviewers R. J. Ferguson and J. T. Etgen are also thanked for their helpful suggestions and comments.

\section{REFERENCES}

Berkhout, A. J., 1984, Seismic resolution: Resolving power of acoustic echo techniques: Geophysical Press Ltd.

Blacquière, G., 1989, 3D wave field extrapolation in seismic depth migration: Ph.D. thesis, Delft University of Technology.

Blacquière, G., Debeye, H. W. J., Wapenaar, C. P. A., and Berkhout, A. J., 1989, 3D table-driven migration: Geophysical Prospecting, 37, 925-958.

Claerbout, J. F., 1985, Imaging the earth's interior: Blackwell Scientific Publications
Etgen, J. T., 1994, Stability of explicit depth extrapolation through laterally-varying media: 64th Annual International Meeting, SEG, Extended Abstracts, 1266-1269.

Gazdag, J., 1978, Wave equation migration with the phase-shift method: Geophysics, 43, 1342-1351.

Gazdag, J., and Sguazzero, P., 1984, Migration of seismic data by phaseshift plus interpolation: Geophysics, 49, 124-131.

Hale, D., 1991a, 3-D depth migration via McClellan transformations: Geophysics, 56, 1778-1785. 1991b, Stable explicit extrapolation of seismic wavefields: Geophysics, 56, 1770-1777.

Holberg, O., 1988, Towards optimum one-way wave propagation: Geophysical Prospecting, 36, 99-114.

McClellan, J. H., 1973, The design of two-dimensional digital filters by transformations: 7th Annual Meeting, Conference on Information Science and Systems, Princeton University, 247-251.

Menke, W., 1989, Geophysical data analysis: Discrete inverse theory: Academic Press Inc.

Nautiyal, A., Gray, S. H., Whitmore, N. D., and Garing, J. D., 1993 Stability versus accuracy for an explicit wavefield extrapolation operator: Geophysics, 58, 277-283.

Paffenholz, J., Stefani, J., McLain, B., and Bishop, K., 2002 SIGSBEE_2A Synthetic subsalt dataset-Image quality as function of migration algorithm and velocity model error: 64th Annual International Meeting, European Association of Geoscience Engineers, Extended Abstracts, B019.

Soubaras, R., 1992, Explicit extrapolation operator synthesis for 2D and 3D migration using the Remez algorithm: 54th Annual International Meeting, European Association of Exploration Geophysicists, Extended Abstracts, 238-239.

Swinnen, G., Wapenaar, K., Thorbecke, J. and Drijkoningen, G., 2001, Design of asymmetric operators using a weighted least-squares approximation: 71st Annual International Meeting, SEG, Expanded proximation: 71st An

Thorbecke, J., 1997, Common focus point technology: Ph.D. thesis, Delft University of Technology.

Thorbecke, J., and Geesink, B., 1999, Performance of 3D depth migration algorithms: 61st Annual International Meeting, European Association of Geoscience Engineers, Extended Abstracts, P130.

Thorbecke, J. W., and Rietveld, W. E. A., 1994, Optimum extrapolation operators-A comparison: 56th Annual International Meeting, European Association of Exploration Geophysicists, Extended Abstracts, P105.

Zhang, J., Verschuur, D. J. and Wapenaar, C. P. A., 2001, Depth migration of shot records in heterogeneous, tranversely isotropic media using optimum explicit operators: Geophysical Prospecting, 49, 287299 